\title{
Contestations et (re)légitimations du renseignement en démocratie \\ Introduction
}

\section{Laurent Bonelli, Hervé Rayner et Bernard Voutat}

\section{OpenEdition \\ Journals}

Édition électronique

URL : https://journals.openedition.org/conflits/20889

DOI : $10.4000 /$ conflits.20889

ISSN : $1777-5345$

Éditeur :

CECLS - Centre d'études sur les conflits - Liberté et sécurité, L'Harmattan

\section{Édition imprimée}

Date de publication : 20 décembre 2019

Pagination : $7-28$

ISBN : 978-2-343-19249-9

ISSN : $1157-996 \mathrm{X}$

Référence électronique

Laurent Bonelli, Hervé Rayner et Bernard Voutat, «Contestations et (re)légitimations du renseignement en démocratie », Cultures \& Conflits [En ligne], 114-115 | été/automne 2019, mis en ligne le 20 décembre 2019, consulté le 03 janvier 2023. URL : http://journals.openedition.org/conflits/ 20889 ; DOI : https://doi.org/10.4000/conflits.20889

\section{(c) $)(1)(9)$}

Creative Commons - Attribution - Pas d'Utilisation Commerciale - Pas de Modification 4.0 International - CC BY-NC-ND 4.0

https://creativecommons.org/licenses/by-nc-nd/4.0/ 


\title{
Contestations et (re)légitimations du renseignement en démocratie
}

\author{
Introduction
}

\section{Laurent BONELLI, Hervé RAYNER et Bernard VOUTAT}

Laurent Bonelli est maitre de conférences en science politique à l'université de Paris-Nanterre et membre de l'Institut des Sciences sociales du Politique (UMR CNRS 7220). Il est co-rédacteur en chef de Cultures \& Conflits et Associate Editor de International Political Sociology. Il a récemment publié, avec Fabien Carrié, La Fabrique de la radicalité. Une sociologie des jeunes djihadistes français (Paris, Le Senil 2018) et, avec Francesco Ragazzi, "La lutte contre la "radicalisation". Genèse et expansion d'un nouveau lieu commun administratif en France et dans l'Union européenne ", Archives de politique criminelle, $n^{\circ} 41$, 2019, pp.119145 .

Hervé Rayner est maître d'enseignement et de recherche en science politique à l'Institut d'Études Politiques (IEP) et du Centre de Recherche sur l'Action Politique de l'Université de Lausanne (CRAPUL). Il a récemment publié, avec Olivier Fillieule et Vanessa Monney, Le métier et la vocation de syndicaliste, l'enquête suisse (Lausanne, Antipodes, 2019), et "L'événement comme forte oscillation des perceptions du possible ", in Amiotte-Suchet L., Salzbrunn M. (dir.), L'événement (im)prévisible : mobilisations politiques et dynamiques religieuses (Paris, Beauchesne, 2019), pp. 79-108.

Bernard Voutat est professeur de science politique a l'Institut d'Études Politiques (IEP) de l'Universite de Lausanne et membre du Centre de Recherche sur l'Action Politique de l'Université de Lausanne (CRAPUL). Ses travaux portent sur la sociologie des institutions politiques et les rapports entre droit et politique. Il a notamment publié, avec Hervé Rayner et Fabien Thetaz, "Les institutions politiques suisses à l'épreuve: le scandale des fiches (1989-1990)", in Mazbouri M., Vallotton F. (dir.), Scandale et Histoire (Lausanne, Antipodes, 2016), pp. 81-98; "L'indignation est-elle un ressort de la scandalisation? Le scandale des fiches en Suisse ", Éthique publique, 2016, 18 (2). 
"Entre ou au-delà des réponses institutionnelles formelles d'une part et de la résistance individuelle d'autre part, il existe bien sûr la possibilité d'une action collective par le biais de groupes de pression, d'organisations non gouvernementales et/ou de mouvements sociaux. Cependant, jusqu'à récemment, peu de travaux ont été consacrés à ces formes de contestations ${ }^{1}$. "

$\mathrm{D}$ ifficile en 2020 d'ignorer le nom d'Edward Snowden, cet ancien contractuel de la National Security Agency (NSA) qui a révélé en 2013 les pratiques d'interception à grande échelle des services de renseignement américains et de certains de leurs homologues occidentaux. L'ampleur planétaire de cette surveillance, attestée par des milliers de documents qu'il a remis à des interlocuteurs choisis (les journalistes Glenn Greenwald et Ewen MacAskill et la documentariste Laura Poitras), a provoqué de larges débats, conférant à ces révélations une magnitude sans commune mesure avec celle des divulgations antérieures ${ }^{2}$. Des milliers d'articles et d'ouvrages ont été écrits, des films grand public et des documentaires réalisés, des chefs d'État se sont publiquement indignés de ces méthodes, des commissions d'enquête parlementaires ont vu le jour, des usagers d'Internet, des défenseurs des libertés publiques se sont mobilisés, des régulations nationales (le Freedom Act de 2015 aux ÉtatsUnis ou la Loi relative au renseignement de 2015 en France par exemple) ou internationales ont été adoptées (comme le Règlement général sur la protection des données [RGPD] de 2016 de l'Union européenne) et de grands opérateurs numériques, comme Google, Yahoo ou Facebook, ont dû revoir certaines de leurs politiques en matière de cryptage des données et de protection de la vie privée (privacy).

Pour autant, cette captation active de données n'a pas cessé. Interpellé par la Chancelière allemande, Angela Merkel - dont un téléphone portable aurait été écouté - le Président des États-Unis, Barack Obama, répondait même un peu abruptement dans une interview donnée le 18 janvier 2014 à la chaîne

1. Benett C., " Privacy Advocates, Privacy Advocacy and the Surveillance Society », in Ball K., Haggerty K. et Lyon D. (dir.), Routledge Handbook of Surveillance Studies, Londres, Routledge, 2012, p. 412.

2. Dès la fin des années 1950, des membres de la NSA tentèrent d'alerter le public sur ses activités. En 1988, le témoignage de l'un d'entre eux permettait au journaliste britannique Duncan Campbell de révéler l'existence du réseau SIGINT Echelon, un vaste programme d'interception des communications privées et publiques mis en place par l'agence et ses alliés. Au début des années 2000, deux anciens employés, William Binney et Thomas Drake avaient, indépendamment l'un de l'autre, dénoncé les pratiques de la NSA. Le premier s'inquiétait de la collecte à grande échelle de données, qui violait selon lui la Constitution des États-Unis; le second du caractère particulièrement intrusif du Trailblazer Project, un programme de captation des communications internet et téléphoniques, lancé à la fin des années 1990. Ces révélations donnèrent lieu à des procès, à des débats parlementaires aux États-Unis et en Europe et connurent un certain écho dans la presse. Néanmoins, les controverses et les mobilisations qu'elles ont suscitées demeurent sans commune mesure avec celles observables dans l'affaire Snowden. Sur cette histoire, voir Delesse C., NSA, Paris, Tallandier, 2016. 
publique $\mathrm{ZDF}$ : « nos agences de renseignement, comme les agences allemandes et toutes les autres, vont continuer à s'intéresser aux intentions des gouvernements de par le monde, cela ne va pas changer. [...] Et ce n'est pas la peine d'avoir un service de renseignement s'il se limite à [collecter] ce qu'on peut lire dans le New York Times ou dans Der Spiegel. La vérité, c'est que par définition le travail du renseignement est de découvrir : que pensent les gens ? que font-ils ? » Ses propos ont le mérite de l'honnêteté, tout comme celui de fragiliser l'antiterrorisme comme principal registre de justification de la surveillance. Ils confirment également que le renseignement constitue une dimension de l'action politique - interne et internationale - à laquelle peu de gouvernants paraissent disposés à renoncer. D’ailleurs, depuis les divulgations de Snowden, les principaux services d'interception tels que la NSA, le Government Communications Headquarters (GCHQ) britannique ou le service technique de la Direction générale de la sécurité extérieure (DGSE) française ont vu leurs budgets et leurs effectifs s'accroître considérablement. Sans que plus grand monde ne semble désormais s'en alarmer.

Cet enchainement de révélations, de scandales, de régulations nouvelles et de continuité des pratiques est particulièrement intéressant pour étudier la question du renseignement. De « l'affaire » Dreyfus à celle du Watergate, en passant par d'autres moins célèbres, des séquences similaires apparaissent en effet avec une étonnante régularité dans le temps et dans l'espace. Elles attestent de la permanence d'activités de renseignement, qui doivent néanmoins se relégitimer périodiquement, notamment lorsqu'elles sont publiquement mises en question. En effet, on aurait tort de penser que la légitimité de la surveillance de groupes et d'individus est acquise une fois pour toutes. L'intensité de cette dernière, ses méthodes et ses cibles font au contraire l'objet d'ajustements fréquents, à la faveur de changements géopolitiques ou technologiques, de modifications des rapports de force entre groupes sociaux ou de transformations des relations à l'intérieur du champ politique ou du champ bureaucratique. Ces variations observables dans l'univers du renseignement impliquent donc de décentrer le regard de sa légitimité vers l'étude de ses processus de légitimation ${ }^{3}$.

\section{Légitimation}

Parler de la légitimité du renseignement comporte en effet un biais normatif. Il s'agirait d'une activité nécessaire au gouvernement, tout à fait acceptable dès lors qu'elle resterait « raisonnable », c'est-à-dire qu'elle porterait sur des groupes «véritablement menaçants » et n'emploierait que les techniques nécessaires pour les neutraliser ou juguler leurs actions. Cette recherche du «bon » renseignement démocratique constitue la ligne de pente suivie par une

3. Lagroye J., «La légitimation », in Grawitz M. et Leca J., (dir.), Traité de science politique, Tome I, Paris, Presses Universitaires de France, 1985, pp. 395-467. 
bonne part des Intelligence Studies qui se sont développées d'abord dans l'espace anglo-saxon ${ }^{4}$ - avec des revues (International Journal of Intelligence and CounterIntelligence et Intelligence and National Security notamment), des sections thématiques permanentes (comme celle de l'International Studies Association) et des départements dans certaines universités, en particulier aux États-Unis ${ }^{5}$ - et plus récemment en Europe continentale ${ }^{6}$. De là, sans doute, les séparations étanches dressées par la plupart des analystes entre les régimes libéraux et ceux qui sont définis comme autoritaires, dont les pratiques sont érigées en contre-modèles 7 .

Il serait bien entendu injuste de résumer en quelques lignes l'ensemble de cette production. Tout au plus peut-on suggérer qu'elle oscille entre un pôle instrumental et un autre plus critique. Les auteurs du premier pôle assimilent surtout les services à un simple outil d'aide à la décision politique, dont ils pointent les «échecs » et auxquels ils proposent des «solutions » pour améliorer, selon les cas, l'analyse, la coopération ou l'efficacité ${ }^{8}$. Ceux du second pôle s'intéressent davantage aux « dérives » ou aux «abus » de certains services au cours de leur histoire et invitent à de nouvelles régulations - politiques et juridiques notamment - afin d'y mettre fin.

On ne contestera pas ici la nécessité et les apports essentiels de ces approches. Elles fournissent des informations précieuses sur les rationalités et les routines - passées et parfois présentes - des services de renseignement, ainsi que sur leur rôle dans les relations internationales ou dans la lutte contre la subversion intérieure. Certains de ces travaux ont également nourri les principaux combats menés au nom des droits humains, par exemple contre l'usage de la torture, les enlèvements de suspects (extraordinary renditions) ou les

4. Voir notamment Ball K., Haggerty K. et Lyon D. (dir.), Routledge Handbook of Surveillance Studies, op. cit. et Gill P. et Phythian M., Intelligence in an Insecure World, Cambridge, Polity Press, 2008.

5. Moran Ch., "Note on Teaching Intelligence », Intelligence and National Security, 31 (1), pp. 118-130.

6. Pour la France, citons notamment Chopin O., Irondelle B. et Malissard A., «Étudier le renseignement en France ", Hérodote, 140, 2011, pp. 91-102 et pour l'Espagne Diaz Matey G., "The Development of Intelligence Studies in Spain », International Journal of Intelligence and CounterIntelligence, 23 (4), 2010, pp. 748-765.

7. Si la coercition apparaît incontestablement plus intense dans les régimes qualifiés d'autoritaires (par le nombre d'individus qu'elle touche et la radicalité des formes qu'elle prend), les méthodes de travail, les modes de raisonnement et les préoccupations de leurs services de renseignement présentent de nombreuses similitudes avec ceux des démocraties libérales. Sur ce point, voir l'ouvrage étonnant de l'anthropologue américaine Katherine Verdery, qui revient, à partir d'archives, sur la surveillance dont elle a fait l'objet de la part des services roumains durant les années 1970-80, alors qu'elle enquêtait sur son terrain. Verdery K., My Life as a Spy: Investigations in a Secret Police File, Londres, Duke University Press, 2018. Pour l'Allemagne de l'Est, voir Combe S., Une société sous surveillance : les intellectuels et la STASI, Paris, Albin Michel, 1999.

8. Johnson L. et Shelton A, «Thoughts on the State of Intelligence Studies: A Survey Report », Intelligence and National Security, 28 (1), 2013, pp. 109-120; Van Puyvelde D. et Curtis S., " "Standing on the Shoulders of Giants": Diversity and Scholarship in Intelligence Studies ", Intelligence and National Security, 37 (7), 2016, pp. 1040-1054. 
détentions illimitées dans des trous noirs judiciaires qui s'étaient multipliés après le 11 septembre 20019 .

Pour autant, l'une et l'autre de ces approches méritent d'être réencastrées dans une sociologie de l'État ou, pour parler comme Max Weber, dans celle d'une «domination en vertu d'une autorité 10 ». En effet, la remarquable permanence d'activités de renseignement à des époques et sous des régimes politiques différents montre qu'elles constituent une dimension essentielle du gouvernement des sociétés complexes. La production de savoirs (sur des populations, des puissances étrangères - alliées ou ennemies - ou des organisations potentiellement concurrentes) représente ainsi une composante fondamentale des relations de pouvoir. À partir de la seconde moitié du XIXe siècle, elle devient une "érudition d'État ", écrit l'anthropologue Alain Dewerpe ${ }^{11}$. Les services de renseignement participent de la sorte à l'immense accumulation de capital informationnel caractéristique du développement et du fonctionnement des États modernes ${ }^{12}$. Mais à la différence de géomètres, de démographes ou de statisticiens engagés dans le même processus, leurs agents ne se contentent pas de construire la réalité sociale par les catégories qu'ils forgent et les découpages qu'ils opèrent. Ils peuvent également agir directement sur celle-ci. Ce sont eux qui habilitent certains acteurs à participer au jeu politique, ou au contraire les disqualifient. Ce sont également eux qui neutralisent, fragilisent ou maintiennent sous contrôle les individus ou les groupes les plus susceptibles de perturber l'ordre social et politique ou de porter atteinte aux « intérêts nationaux ». Ils apparaissent ainsi simultanément comme des vecteurs de la violence symbolique de cet « appareil de domination spécialisé ${ }^{13}$ » qu'est l'État, mais également de sa violence physique. Cette double dimension explique sans doute la récurrence des controverses qui les frappent. Et c'est ici que le détour par les contestations constitue une entrée utile en ce qu'elles rendent visibles des processus de légitimation qui habituellement restent de l'ordre de l'implicite.

\section{Contestations}

Les conjonctures au cours desquelles les services de renseignement sont mis en cause - parfois dans leur existence même - dans les champs politique, médiatique, judiciaire (des tribunaux nationaux à la Cour européenne des droits de l'homme), ou même dans la rue, ne font l'objet que d'un traitement

9. Pour une synthèse, voir Guild E., Bigo D. et Gibney M., Extraordinary Rendition. Addressing the Challenges of Accountability, Londres, Routledge, 2018.

10. Weber M., La domination, Paris, La Découverte, 2013, p. 45.

11. Dewerpe A., Espion. Une anthropologie historique du secret d'État contemporain, Paris, Gallimard, 1994, pp. 219 et suiv.

12. Bourdieu P., Sur l'État : Cours an Collège de France (1989-1992), Paris, Seuil, 2012, p. 336 ; Desrosières A., La politique des grands nombres. Histoire de la raison statistique, Paris, La Découverte, 2000.

13. Elias N., La dynamique de l’Occident, Paris, Calmann-Lévy, 1990, p. 26. 
marginal dans les Intelligence Studies. Quoique signalées dans plusieurs travaux (sous les expressions " polémiques », « affaires », " manifestations d'opposition ", "vives inquiétudes », " vigoureuses campagnes de protestation », " résistances »), ces contestations demeurent mentionnées de manière très allusive ${ }^{14}$. Il y a là une lacune ${ }^{15}$, d'autant que ces contestations révèlent à la fois les contraintes de légitimation auxquelles sont soumis ces services spécialisés et les conditions de possibilité de leur remise en question.

Une telle perspective impose toutefois de ne pas confondre les scandales, c'est-à-dire les mobilisations multisectorielles autour d'une dénonciation ${ }^{16}$, et les faits présumés scandaleux invoqués par les dénonciateurs. Elle invite à faire dialoguer la sociologie des mobilisations et la sociologie des institutions, et en l'espèce les travaux sur les scandales et ceux sur le renseignement. D'une part, la prise de conscience de l'étendue de la surveillance provient en grande partie de « révélations » afférentes à des scandales. De l'autre, une dimension récurrente et officieuse du renseignement (interne et externe) consiste de longue date à désamorcer, ou au contraire à fomenter, des scandales.

Ces phases critiques permettent tout d'abord de révéler certaines propriétés du renseignement, éclairant à la fois la fréquence des dénonciations, mais aussi leur caractère souvent éphémère. Ainsi en est-il d'une propriété essentielle, celle du secret ou tout au moins du flou qui entoure les pratiques en question. Cette dimension restreint sans doute la jouabilité des dénonciations d'activités difficilement atteignables par la critique du fait de leur dissimulation et des protections $-\mathrm{y}$ compris légales - dont elles jouissent.

Paradoxalement, les groupes et individus qui en sont les cibles privilégiées - qui en ont donc une expérience concrète - apparaissent souvent bien mal placés pour les mettre en cause. Certes, ils déplorent inlassablement dans des publications en affinité avec leur cause, devant des magistrats ou dans la presse, les abus dont ils s'estiment victimes. Ils invoquent fréquemment des mauvais traitements (lors d'interrogatoires par exemple), des limitations de circulation (les no fly lists, les interdictions de quitter le territoire ou les assignations à résidence notamment), des mesures perturbant leur activité (comme le gel des avoirs financiers ou la fermeture administrative de locaux)

14. À la différence des Surveillance Studies, qui analysent, en référence à Michel de Certeau et à James Scott, des formes individualisées de « résistance au quotidien » afin de caractériser les relations entre surveillants et surveillés, on trouve surtout des travaux prenant pour objet des mouvements en faveur de la protection de la vie privée ou/et de l'éthique des droits de l'homme et de l'État de droit. A contrario, on lira avec intérêt Amicelle A. et FavarelGarrigues G., «La lutte contre l'argent sale au prisme des libertés fondamentales : quelles mobilisations ? », Cultures E Conflits, n $^{\circ} 76,2009$, pp. 39-66.

15. Piazza P., «Les résistances au projet INES », Cultures $E$ Conflits, 64, 2007, pp. 65-75; Benett C., «Privacy Advocates, Privacy Advocacy and the Surveillance Society », op. cit.

16. Rayner H., Dynamique du scandale, de l'affaire Dreyfus à Clearstream, Paris, Le Cavalier Bleu, 2007. 
ou la sévérité des peines auxquelles ils sont condamnés. Mais s'ils obtiennent parfois des réparations individuelles 17 et plus rarement collectives ${ }^{18}$, leurs allégations ne suffisent pas à miner significativement la légitimité des actions des services de renseignement. Tout se passe comme si leur caractère minoritaire, comme la virulence de leur contestation de l'ordre politique et social, rendait difficile l'expression d'une solidarité plus large. Ceux qui sont le plus directement concernés par les pratiques les plus coercitives des services sont donc à l'évidence les moins à même de les contester avec efficacité. Les expressions de «terroristes » ou de " radicaux» (et plus caricaturalement celles de "fous de dieu », «fanatiques » ou de «bouchers sanguinaires ») par lesquelles ils sont publiquement définis contribuent à les présenter sous l'angle d'une altérité irréductible qui les écarte a priori de tout débat sur les formes de contrôle acceptables dans la société. Au nom de la défense de celle-ci-pour paraphraser Michel Foucault - des formes d'exceptionnalisme seraient admissibles les concernant ${ }^{19}$.

A contrario, c'est lorsque les pratiques de contrôle et de surveillance semblent plus indiscriminées, lorsqu'elles paraissent - potentiellement au moins pouvoir toucher des secteurs beaucoup plus larges de la population, qu'elles ouvrent la possibilité d'une remise en cause. "L'affaire de Tarnac », en France, est intéressante sous ce rapport. Neufs jeunes gens, suspectés d'avoir saboté des caténaires de lignes de chemin de fer en novembre 2008, ont été mis en examen pour association de malfaiteurs en relation avec une entreprise terroriste, sur la foi de la surveillance intensive d'agents de renseignement ${ }^{20}$. Ce cas

17. En juillet 2012, la CEDH a par exemple ordonné la remise en liberté de la militante d'ETA Inés del Rio Prada, maintenue en détention au-delà de sa durée légale, et condamné le gouvernement espagnol à lui verser 30000 euros pour « dommage moral ». De la même manière, Omar Khadr, un jeune Canadien capturé en Afghanistan et détenu pendant plus de dix ans à Guantanamo, a reçu les excuses de son gouvernement et une compensation de plusieurs millions de dollars pour les mauvais traitements endurés durant sa captivité. Néanmoins, ces situations demeurent exceptionnelles et la plupart des requêtes sont rejetées, y compris par les Cours de justice.

18. Par exemple, l'Organisation des Moudjahidines du peuple iranien (OMPI), placée sur la liste des « organisations terroristes » des États-Unis et de l'Union européenne, est l'une des rares a en avoir été retirée à l'issue d'une vaste campagne de mobilisation internationale à la fois politique et juridique. Sa position d'opposante au régime iranien, dans un contexte de durcissement de celui-ci après le soulèvement de 2009 (mouvement vert), comme la prise de distance de l'organisation vis-à-vis des actions armées, expliquent sans doute cette trajectoire singulière.

19. Les débats autour du controversé droit pénal de l'ennemi (Feindstrafrecht), théorisé par le juriste allemand Günter Jakobs et qui prive les individus « déloyaux » politiquement des protections du droit dont peuvent bénéficier les autres citoyens, vont en ce sens. La catégorie "d'ennemi combattant », inventée par l'administration américaine après le 11 septembre 2001, et qui place ceux auxquels elle s'applique en dehors du droit pénal interne et en dehors du droit international constitue sans doute l'une des réalisations les plus abouties de ce mouvement. Voir Jakobs G., "On the Theory of Enemy Criminal Law », in Dubber M. (dir.), Foundational Texts in Modern Criminal Law, Oxford, Oxford University Press 2014, pp. 415-424. Pour une critique, voir notamment le numéro spécial de la Revue de science criminelle et de droit pénal comparé, intitulé « Droit pénal de l'ennemi - Droit pénal de l'inhumain » (2009-01/03). 
fut présenté par la ministre de l'Intérieur de l'époque comme la preuve d'une résurgence d'une extrême gauche (rebaptisée à cette occasion « anarcho-autonome ») prête à passer à l'action violente pour déstabiliser l'ordre établi. La défense des prévenus fit capoter cette tentative de labellisation. Dans des tribunes, des analyses, comme lors de leurs interrogatoires par les magistrats ou lors de leur procès, les prévenus n'ont eu de cesse de questionner les méthodes des policiers et de pointer les incohérences dans les récits construits pour démontrer leur dangerosité. L'antiterrorisme, écrivent-ils, " a non seulement l'art de faire passer des chihuahuas pour des loups, mais en outre celui de faire taire toute protestation à son sujet 21 ». Dès lors, sans abandonner un combat opiniâtre sur les pièces de leur dossier, ils se sont efforcés d'élargir la critique à sa fonctionnalité. Ils arguent ainsi qu'il ne ciblerait pas « centralement ceux sur qui il s'abat, mais l'ensemble de la population ; qu'il n'[est] donc pas une procédure judiciaire, mais un mode de gouvernement 22 ». En pointant le déséquilibre apparent entre le caractère relativement bénin des faits qui leur étaient reprochés et l'ampleur de la réaction institutionnelle (tant policière que judiciaire), ils ont pu avec un certain succès contester des pratiques pourtant banales en la matière et porter le débat sur le terrain de la subversion politique acceptable en démocratie. Servis par des propriétés particulières - un capital culturel qui leur permettait de formaliser un contre-récit, l'usage de catégories de pensée issues d'une longue histoire des luttes sociales et politiques en Europe, un accès préalable à la sphère éditoriale - ils ont pu publiciser leur cause (y compris dans les médias dominants) et rallier des soutiens, bien audelà des milieux les plus militants. À telle enseigne que la qualification « terroriste » fut finalement abandonnée par les magistrats instructeurs et qu'au terme de dix ans de procédures judiciaires, ils furent finalement relaxés pour les dégradations dont ils étaient accusés. Plus largement, cette « affaire » représente désormais un souvenir douloureux pour nombre de professionnels de l'antiterrorisme, peu habitués à voir leurs méthodes et leurs rationalités disséquées publiquement.

Pour autant, cet exemple réussi de contestation n'a pas produit d'aggiornamento dans le rôle des services de renseignement, ni même dans leurs logiques de fonctionnement. Celles-ci continuent à se déployer à l'identique par exemple dans la lutte contre la violence politique à référence islamique, cette fois-ci sans rencontrer d'opposition ${ }^{23}$. L'inégale capacité des groupes et des individus à se défaire de l'étiquette stigmatisante qui leur est apposée et à présenter la surveillance comme une menace pour de larges secteurs de la

20. Sur cette affaire, voir la belle enquête du journaliste David Dufresne : Tarnac, magasin général, Calmann-Lévy, Paris, 2012.

21. Le Monde, 14 février 2014.

22. Ibid.

23. Comme en attestent les procès des individus qui sont allés combattre en Syrie et en Irak (ou ont souhaité le faire). Voir Mégie A., " Le contentieux judiciaire antiterroriste depuis 2015 en France : "massification", spécialisation et politisation” », in Séze R. (dir.), Les États européens face aux militantismes violents, Paris Riveneuve, 2019, pp. 187-206. 
population constitue sans doute l'une des conditions de possibilité de mobilisations élargies. Mais une remise en question plus profonde des services de renseignement nécessite d'autres facteurs, parmi lesquels la défection de certains groupes - dans l'État notamment - qui leur apportaient jusque-là leur soutien ou se contentaient de fermer les yeux.

Le politiste Michel Dobry analyse ainsi les scandales qui les affectent régulièrement comme l'un des marqueurs les plus visibles du délitement des relations ordinaires qu'ils entretiennent avec les autres secteurs stratégiques de nos systèmes politiques ${ }^{24}$. Celles-ci sont en fait assez spécifiques. Les services doivent fournir des informations considérées comme «pertinentes » par les gouvernants (tant en matière intérieure que pour les relations internationales), à l'aune desquelles ces derniers vont jauger leurs performances et leur « utilité ». Les représentants des autorités renoncent de leur côté au contrôle au jour le jour des activités de renseignement et acceptent leur opacité. Ceci leur permet notamment de ne pas être impliqués dans des intrigues qu'il vaut mieux ne pas connaître ou feindre d'ignorer pour être en mesure de s'en désolidariser dans les moments où, pour des raisons contextuelles, la contestation est soudain perçue comme plus jouable que d'ordinaire. C'est ce que le criminologue canadien Jean-Paul Brodeur appelait « le chèque en gris » signé par les dirigeants en faveur des services spécialisés ${ }^{25}$. Alain Chouet, un ancien chef de service de la DGSE, résume ce lien particulier de manière un peu provocatrice : «tous les États du monde disposent en quantité souvent non négligeable de magistrats, de policiers, de diplomates, de militaires, d'experts en tout genre, pour concevoir et mettre en œuvre leurs décisions politiques légales. Si à côté de ces dispositifs, les mêmes États entretiennent souvent à grands frais des services spéciaux, ce n'est pas pour faire double emploi avec les autres, ou organiser une saine émulation sportive entre fonctionnaires. C'est pour pouvoir s'affranchir, à l'occasion et s'il en est besoin, de la légalité intérieure ou extérieure ou de leurs engagements internationaux et, de préférence, sans se faire prendre la main dans le sac, ce qui nécessite une certaine technicité. C'est donc ne rien comprendre que d'accuser les services secrets de faire “dans l'illégalité”. Bien sûr, qu'ils font “dans l'illégalité”. Ils ne font même que cela. C'est leur vocation et leur raison d'être 26 ».

Ces relations durables, que Dobry qualifie de «transactions collusives » parce qu'elles ne sont jamais complètement avouables, constituent sans doute l'un des principaux fondements de la légitimité des services de renseignement. Aussi n'est-il pas surprenant que celle-ci soit périodiquement mise en cause lors de bouleversements politiques, lorsque certaines collusions ne vont plus

24. Dobry M., «Le renseignement dans les démocraties occidentales. Quelques pistes pour l'identification d'un objet flou ", Cabiers de la sécurité intérieure, 30, 1997, p. 74.

25. Brodeur J-P., "High Policing and Low Policing: Remarks about the Policing of Political Activities », Social Problems, vol. 30, 5, 1983, pp. 507-520.

26. Chouet A., La sagesse de l'espion, L'œil neuf éditions, Paris, 2010, pp. 15-16. 
de soi. Les exemples foisonnent dans l'histoire de remises en question plus ou moins radicales de services liées à des alternances ou à des changements de régime ${ }^{27}$. Mais les critiques peuvent également venir d'autres administrations, qui font valoir qu'elles sont plus compétentes ou plus efficaces dans tel ou tel domaine. Ainsi, on ne comprendrait pas le discrédit - relatif - de la CIA sous l'administration Obama sans voir le rôle joué par le FBI dans la condamnation des pratiques de torture, d'enlèvement et de détention illimitée auxquelles l'agence avait recours dans le cadre de la guerre globale contre la terreur. Parfois, ce sont des dissensions internes aux services - à la faveur d'une transformation du recrutement, liée par exemple à des mutations technologiques qui sapent les modus vivendi établis antérieurement. Et c'est ici que l'on perçoit l'utilité de la sociologie des scandales. Ceux qui «prennent»en se déployant dans et entre plusieurs espaces sociaux résultent et impulsent des mobilisations hétérogènes, à la fois extérieures et intérieures à l'État, dont il faut à chaque fois retracer les raisons, les temporalités et les enchaînements. Ce type de concaténation expose les professionnels de la surveillance à de sérieuses incertitudes, les mobilisations multisectorielles et les fortes oscillations des perceptions du possible dont les scandales sont faits ayant pour propriété de faciliter les dénonciations et les lâchages 28 .

\section{Relégitimation}

Dans la mesure où elles appellent des ripostes institutionnelles visant à désamorcer les contestations, les crises que traverse le renseignement donnent plus facilement à voir les ressorts de sa légitimation. Avec des intensités et selon des modalités variables, cette activité a connu, souvent sous l'emprise de scandales, de nombreuses réformes dans les démocraties libérales depuis les années 1990. Celles-ci se caractérisent notamment par des velléités de contrôle plus conséquentes au regard de la période précédente, celle de la guerre froide, où les services conservaient une forte autonomie par rapport aux autorités politiques et disposaient d'une large marge de manœuvre d'un point de vue légal.

Ce mouvement, qui façonne certaines contraintes pesant sur les professionnels, repose de plus en plus sur des normes légales censées délimiter les pratiques admissibles, définir leurs finalités (conçues par exemple par le droit interne en termes de police "préventive " par opposition aux tâches dites répressives de la police judiciaire) et fixer leurs modalités (identification des

27. Il reste néanmoins fréquent que les nouveaux gouvernants changent d'avis en découvrant l'intérêt de ces services au moment de leur accession au pouvoir. En France, l'exemple des républicains des débuts de la III République ou celui des socialistes avant 1981 sont connus. On trouvera un autre exemple de ces continuités - y compris postcoloniales - au Tchad dans l'article de Bat J-P., Duranton A., El Ghaziri S., Sigalas M. et Stemmelin M., « Renseigner et administrer la terreur sous Hissein Habré : la Direction de la documentation et de la sécurité », Champ pénal/Penal field, 17, 2019, [En ligne], consulté le 25 novembre 2019.

28. Rayner H., « De quoi les scandales sont-ils faits ? ", Traverse, 3, 2015, pp. 33-45. 
personnes, établissement de fichiers, infiltrations, suivi des transactions financières, écoutes téléphoniques, contrôle des échanges numériques, etc.). À cette codification des pratiques par le droit s'ajoutent des mécanismes de contrôle politique, parlementaire, judiciaire ou administratif supposés garantir le fonctionnement «démocratique » des services de renseignement ${ }^{29}$. Des propos comme ceux d'Alain Chouet sur « l'illégalité » constitutive de ces derniers seraient désormais plus difficilement tenables. Ils cèdent plutôt la place à des discours en termes de dérogations au droit commun, qui ne seraient toutefois pas discrétionnaires, étant soumises à des supervisions extérieures, même si celles-ci ne sont pas publiques. Toute une littérature se développe depuis lors, qui insiste sur la tension (ou la contradiction) entre une « raison d'État » associée aux pratiques de renseignement et les libertés fondamentales reconnues par les démocraties libérales, ainsi que par des traités internationaux, comme la Convention Européenne des Droits de l'Homme (CEDH) ${ }^{30}$. L'expertise juridique imprègne désormais la teneur des controverses. Le droit devient un terrain d'affrontements où s'opposent des gouvernants, soucieux d'asseoir la légitimité du renseignement en l'inscrivant dans les principes de l'État de droit, et des acteurs (souvent des ONG actives dans ce domaine, comme Privacy International, Human Rights Watch ou Amnesty International, parfois des juristes de grandes compagnies comme Microsoft, Yahoo ou Apple) mobilisés contre les « dérives » ou « excès » des services spéciaux au nom de la défense des droits fondamentaux (libertés publiques, droit à la vie privée, protection des données, publicité des actes étatiques), voire de leurs intérêts commerciaux ${ }^{31}$.

Ce déplacement invite à interroger ces dispositifs juridiques sous l'angle de la force ambivalente du droit, instrument de légitimation du renseignement d'un côté, moyen permettant de le dénoncer de l'autre. On observe en effet un activisme judiciaire significatif de la part de défenseurs des libertés auprès des cours nationales et surtout internationales en charge de la protection des droits humains. Cette évolution suscite d'ailleurs un intérêt grandissant des sciences sociales, en particulier de la sociologie des mouvements sociaux et de

29. Bigo D., "Security, surveillance and democracy », in Ball K., Haggerty K. et Lyon D., The International Handbook of Surveillance Studies, op.cit., pp. 277-284 ; Abu-Laban Y., "The Politics of Surveillance: Civil Liberties, Rights and Ethics ", ibid., pp. 420-427.

30. Gill P., "Security Intelligence and Human Rights: Illuminating the 'Heart of Darkness?' », Intelligence and National Security, 2009, 24:1, pp. 78-102 ; Preuss-Laussinotte S., «Bases de données personnelles et politiques de securite : une protection illusoire ? ", Cultures E Conflits, 2006, n64, pp. 77-95 ; "L’Union européenne et les technologies de sécurité », ibid., pp. 97-108; «L'élargissement problématique de l'accès aux bases de données européennes en matière de sécurité ", Cultures E Conflits, ${ }^{\circ}$ 74, 2009, pp. 81-90. Dans le domaine du terrorisme, voir Saulnier-Cassia E. (dir.), La lutte contre le terrorisme dans le droit et la jurisprudence de l'Union européenne, Paris, LGDJ, 2014.

31. On trouvera une présentation synthétique de plusieurs cas récents concernant la collecte de données numériques dans Sigint intelligence transnational activities in France and Europe. Report, pp.16-28, (https://www.sciencespo.fr/ceri/sites/sciencespo.fr.ceri/files/UTIC6Hoffmann_et_Michalon_Compte-rendu_de_colloque_SIGINT_(english).pdf), consulté le 24 novembre 2019. 
l'action collective, dans le cadre de travaux portant sur les usages militants du droit. Arme à double tranchant ${ }^{32}$, tantôt défensive lorsque le non-respect du droit est invoqué comme un «bouclier », tantôt offensive, lorsqu'elle est brandie comme un levier « cognitif » permettant de monter en généralité en vue de formuler un grief et de nommer une injustice ${ }^{33}$, le droit devient un terrain que les ONG investissent plus volontiers. Pouvant parfois se prévaloir de ressources juridiques particulières, elles s'appuient également sur une expertise plus large (sociologique, anthropologique ou historique) pour faire avancer leur cause au niveau transnational dans le domaine des droits humains ${ }^{34}$, et en particulier pour contester les législations nationales encadrant les activités de renseignement, comme l'atteste l'importance croissante du contentieux dans ce domaine. Les litiges judiciaires offrent sans doute des opportunités importantes aux contestataires, qui peuvent ainsi, à partir de situations particulières, dénoncer publiquement certaines pratiques et revendiquer des changements de législation au nom de valeurs ou principes fondamentaux. Ces formes de guérilla juridique exercent une pression sur les cours, qui reconnaissent parfois certains griefs, ainsi que sur les gouvernements et les services de renseignement, remis en cause à plusieurs reprises par des jugements 35 .

Cependant, ces usages contestataires du droit se heurtent à de nombreux obstacles. Les procédures sont longues, complexes et difficiles d'accès pour les ONG et a fortiori pour les particuliers : on pense notamment à la règle en vigueur devant la $\mathrm{CEDH}$, qui impose un épuisement des voies de recours nationales avant l'examen des saisines, décourageant ainsi nombre de requérants et de litiges ${ }^{36}$. À quoi s'ajoutent la sophistication croissante du droit dans ce domaine et les ripostes des gouvernements visant à faire admettre, bénéficiant du flou entourant les notions juridiques, certaines restrictions aux

32. Israël L., L'arme du droit, Paris, Presses de Sciences Po, 2009 ; Mc Cann M.-W., « Law and Social Movements : Contemporary Perspectives ", Annual Review of Law and Social Science, $\mathrm{n}^{\circ}$ 2, 2006, p.17-38; Agrikoliansky E., «Les usages protestataires du droit », in Agrikoliansky E., Sommier I. et Fillieule O., Penser les mouvements sociaux. Conflits sociaux et contestations dans les sociétés contemporaines, Paris, La Découverte, 2010.

33. Felstiner W.-L. F., Abel R.-L. et Sarat A., « The Emergence and Transformation of Disputes: Naming, Blaming, Claiming... », Law E Society Review, vol. 15, n³/4, 1980, pp. 631-654.

34. Ringelheim J., "Le role des ONG dans le contentieux international des droits de l'homme », Journal européen des droits de l'homme-European Journal of Human Rights, 2018/2, pp. 7183 et «Les droits humains saisis par les mouvements sociaux : introduction ", Revue interdisciplinaire d'études juridiques, vol. 75, $\mathrm{n}^{\circ} 2$ 2, 2015, pp. 59-66 ; Pieret, J., «Étudier les droits humains pour mieux comprendre les mouvements sociaux ? ", Revne interdisciplinaire d'études juridiques, vol. 75, $\mathrm{n}^{\circ} 2,2015$, pp. 167-188.

35. Par exemple, le Investigatory Powers Act adopté au Royaume Uni de novembre 2016 - baptisé « loi des fouineurs » par ses détracteurs - fut finalement déclaré contraire aux législations européennes par la UK High Court en avril 2018, au terme d'une virulente mobilisation juridique, portée en autres par l'organisation Liberty. Il fut amendé par le gouvernement et le texte qui lui succéda (Data Retention and Acquisition Regulations 2018) comporte des limitations sur la surveillance qui n'existaient pas dans le texte original.

36. Preuss-Laussinotte S., "Technologies de sécurité et respect des droits et libertés individuelles », in Agrouhm C. et al., Identification et surveillance des individus : Quels enjeux pour nos democraties ?, Paris, Éditions de la Bibliothèque publique d'information/Centre Pompidou, 2010, pp. 62-69. 
droits fondamentaux afin de faire prévaloir, par exemple, les impératifs de la lutte contre le terrorisme ou du contrôle des migrations ${ }^{37}$.

Cette configuration relativement nouvelle témoigne d'un processus plus large de juridicisation de nos sociétés, un phénomène " polymorphe 38 » qui désigne tout à la fois l'encadrement toujours plus important des rapports sociaux par le droit, le rôle croissant des instances judiciaires dans la détermination de l'action publique et l'emprise grandissante des catégories juridiques dans la perception et la construction des enjeux politiques. Cependant, pour justifiés que soient les débats essentiellement normatifs que suscite l'expertise juridique à propos de la légitimité du renseignement et la contrainte de mise en cohérence de cette activité avec l'ordre juridique des États démocratiques, il convient de ne pas céder à une « conception idéalisée du droit » fondée sur le « fétichisme de la règle 39 », forme de croyance en sa force intrinsèque que les juristes seraient à même de faire advenir au nom de la raison juridique dont ils se revendiquent et qu'ils pensent incarner. Comme le souligne le politiste Bernard Lacroix, les énoncés juridiques sont loin de posséder dans les faits la force contraignante qu'on leur prête habituellement : « Le respect de la règle pourrait bien n'être qu'une des modalités parmi bien d'autres dans l'ensemble de ses usages éventuels 40 ». S'il est vrai, poursuit-il, que « l'argument juridique tend à irriguer, aujourd'hui peut-être plus qu'en d'autres circonstances, la vie politique officielle [...], il n'en reste pas moins que le recours à la casuistique et à l'herméneutique juridiques n'est pas d'un grand secours pour comprendre la construction et la dynamique des institutions 41 », et pour nous, les activités de renseignement telles que pratiquées par les services.

Certes, l'abondante jurisprudence des cours nationales et internationales joue un rôle majeur dans la détermination «sur le papier » des pratiques autorisées. Dans certains cas, elle peut même avoir des incidences pratiques importantes sur la liberté d'action dont les services de renseignement estiment pouvoir disposer. Toutefois, phénomène ambivalent, la juridicisation du renseignement contribue aussi à sa légitimation. En exerçant une contrainte sur des services désormais sommés d'agir dans les règles, le droit constitue alors un puissant vecteur de normalisation de leurs pratiques. Au point que certains

37. Sur tous ces points, voir Ringelheim J., « Legalizing Human Rights: Modest Reflections on the Limits and Flipsides of a Legal Ideal », in Hoc A., Wattier S. et Willems G. (dir.), Human Rights as a Basis for Reevaluating and Reconstructing the Law, Bruxelles, Bruylant, 2016, pp. 13-27.

38. Commaille J., Dumoulin L., « Heurs et malheurs de la légalité dans les sociétés contemporaines. Une sociologie politique de la judiciarisation ", L'Année sociologique, 2009, 59 (1), pp. 63-107.

39. Chevallier J., L'État de droit, Paris, Montchestien, 2017 (6e éd.).

40. Lacroix B., "Le politiste et l'analyse des institutions », in Lacroix B. et Lagroye J. (dir.), Le président de la République. Usages et genèses d'une institution, Paris, Presses de la FNSP, 1992, p. 18.

41. Ibid., p. 71. 
responsables en viennent eux-mêmes à demander la régulation par la loi de leurs activités. Ainsi en est-il de Patrick Calvar, directeur central du renseignement intérieur français, qui réclamait une loi-cadre devant le parlement : « Je trouve l'approche anglo-saxonne très pragmatique. S'en inspirer permettrait d'avoir des règles claires quant à nos possibilités légales d'action, puis le contrôle a posteriori permet de vérifier si les actes effectués respectaient bien le cadre légal. Or, nous sommes dans une "zone grise” qui rend l'action difficile. Nous avons besoin de missions définies, de moyens adaptés, de pouvoirs juridiquement reconnus et de contrôles 42 ».

On le voit à travers cet exemple, la légalisation de certaines pratiques du renseignement (que les intéressés aiment, avec un beau sens de l'euphémisme, appeler alégales) n'est pas seulement imputable à des contraintes de légitimation relevant d'abord de considérations " normatives 43 » liées à l'État de droit et au respect des droits fondamentaux. Elle doit aussi se comprendre à partir de la place que prend le droit dans l'ordinaire de la plupart des services de l'État, dans le domaine du renseignement comme ailleurs. Soucieux de préserver leurs prérogatives au nom du droit et d'être en mesure de s'en prévaloir dans les moments de crise ou de remise en cause, les professionnels du renseignement s'appuient aussi sur le droit dans les transactions avec d'autres acteurs (autorités politiques, médias, parlementaires, partis, entreprises, etc.), notamment dans des conjonctures où leurs activités, voire leur existence même, sont contestées. De ce point de vue, il n'y a pas lieu de considérer que leur rapport au droit, à tous les niveaux hiérarchiques, soit fondamentalement différent de celui qui caractérise les agents de l'État d'une manière générale. À l'image des fonctionnaires appliquant les politiques locales du logement ${ }^{44}$, les agents de renseignement ne sauraient être considérés comme de purs exécutants des règles encadrant leurs activités, tant il est vrai que « le droit n'est pas fait pour être appliqué mais pour orienter les comportements 45 ». Cette perspective implique alors de déplacer le regard de la règle vers les pratiques, ou plus précisément vers les usages que les agents du renseignement en font, sachant que, comme le souligne Pierre Bourdieu s'inspirant de Max Weber, « on obéit à la règle lorsque l'intérêt à lui obéir l'emporte sur l'intérêt à lui désobéir 46 ». Considérant la plasticité interprétative des législations portant sur le renseignement, notamment en ce qui concerne leur dépendance à l'égard des normes générales et abstraites relevant de la protection des droits fondamen-

42. Audition devant la Commission des lois constitutionnelles, de la législation et de l'administration générale de la République, 30 octobre 2013, p.10. Cette loi verra le jour deux ans plus tard (Loi n ${ }^{\circ} 2015-912$ du 24 juillet 2015 relative au renseignement).

43. Comme l'indique opportunément Michel Dobry dans « Le renseignement dans les démocraties occidentales...», art. cit., p. 55.

44. Bourdieu P., « Droit et passe- droit. Le champ des pouvoirs territoriaux et la mise en œuvre des règlements ", Actes de la recherche en sciences sociales, $\mathrm{n}^{\circ}$ 81/82, mars 1990, pp. 86-96.

45. Lascoumes P. et Le Bourhis J-P., « Des "passe-droits" aux passes du droit. La mise en œuvre socio-juridique de l'action publique », Droit et Société, 32, 1996, pp. 51-73.

46. Bourdieu P., « Droit et passe-droit... », art. cit., p. 87. 
taux, on comprend ainsi mieux que les services de renseignement puissent faire autre chose que de simplement se soumettre aux règles officiellement conçues pour les « encadrer ». Ils disposent pour cela d'une marge de manœuvre importante, du reste reconnue par la loi aussi bien que par la jurisprudence, notamment en ce qui concerne le secret entourant leurs pratiques.

Il faut en effet être habilité pour accéder à certaines informations et ce n'est pas toujours suffisant. "Le besoin d'en connaître ", une formule répétée à satiété dans cet univers, restreint encore l'accès à celles jugées strictement nécessaires à l'accomplissement d'une mission. Le secret et le cloisonnement sont justifiés par la nécessité de protéger les agents de renseignement, leurs sources et leurs méthodes, du regard de leurs adversaires. Ils incitent à la loyauté des agents vis-à-vis de l'institution sous l'effet de sanctions en cas de non-respect. En outre, ils renforcent leur position dans les champs bureaucratique et politique, tout en les mettant à l'abri d'un questionnement trop précis des magistrats, des élus et, bien sûr, des journalistes ou des chercheurs. " Le secret, écrivait déjà Georg Simmel, place la personne dans une situation d'exception, il agit comme un charme dont la détermination est purement sociale, indépendant dans son principe du contenu qui protège ; mais naturellement, ce charme croît dans la mesure où le secret que l'on détient en exclusivité est important et vaste 47 ». Le secret apparaît ainsi comme une ressource rare, puisqu'il fonctionne comme un argument d'autorité difficilement contestable par ceux qui n'y ont pas accès. "L'habilitation est une onction » rappelle Dewerpe ${ }^{48}$. Que répondre à des assertions du type "je sais des choses que vous ne savez pas et que je ne peux pas vous dire » ? Cette revendication d'un savoir secret accroît donc la force au jeu des services de renseignement, tout en soustrayant les aspects les plus pratiques de leur travail de possibles critiques ou évaluations externes. La nécessité de cette protection opérationnelle ouvre alors la porte à un usage plus stratégique du secret. Commentant à des journalistes les politiques strictes de classification des missions, des locaux et des personnels du renseignement intérieur français après 2008, un agent explique : « initialement, le secret défense était censé empêcher l'infiltration par le KGB, aujourd'hui, il permet surtout d'opacifier la boutique en mettant des vitres fumées partout 49 ». L'un de ses homologues de la DGSE décrit pour sa part les formes de contrôle qu'il considère acceptables en ces termes : « bien sûr, il ne peut y avoir de transparence, mais il peut y avoir de la translucidité. Il faut laisser voir ce qui se passe, sans montrer le détail » (notes de terrain novembre 2018).

47. Simmel G., Secret et sociétés secrètes, Strasbourg, Éditions Circé, 1996, p. 43.

48. Dewerpe A., Espion..., op. cit., p. 380.

49. Cité dans Recasens O., Hassoux D. et Labbé Ch., L'espion du président. Au coeur de la police politique de Sarkozy, Paris, Robert Laffont, 2012, p. 153. 
On mesure dans ces propos les arrangements qui peuvent exister au quotidien avec le droit interne ou international. Ici peut être plus qu'ailleurs, les professionnels « servent la règle autant qu'ils s'en servent », " la mobilisent et l'utilisent comme une arme » 50 à l'égard de l'ensemble des acteurs (dénonciateurs compris) impliqués à un titre ou à un autre dans le renseignement. La maxime de Bourdieu selon laquelle « le jeu avec la règle fait partie de la règle $\mathrm{du}$ jeu 51 » s'applique sans doute tout particulièrement au domaine du renseignement. Et les controverses que ce dernier suscite de manière récurrente, qui se situent pour beaucoup d'entre elles sur le terrain du droit, se trouvent ainsi circonscrites à des oppositions sur les limites des pratiques admissibles en regard des « dérapages », " abus » et autres « excès » de services aux objectifs désormais normalisés. C’est probablement aussi dans cette perspective qu'il convient d'appréhender les nouvelles latitudes garanties par les réformes successives des législations sur le renseignement dans les démocraties libérales. Tout se passe comme si les controverses juridiques, dans le contexte d'un rapport de force très inégal entre gouvernants et gouvernés, avaient la plupart du temps pour effet d'ouvrir en les légalisant les marges de manœuvre des services de renseignement lorsqu'elles sont remises en cause ${ }^{52}$.

\section{Croiser les questionnements et les terrains dans le temps et dans l'espace}

Les articles présentés dans cette livraison de Cultures $\varepsilon$ Conflits résultent d'une réflexion collective sur le renseignement issue de la rencontre entre des chercheurs engagés dans l'ANR UTIC (Usages des Technologies liées à l'Interception des Communications) 53 et des chercheurs de la Faculté de Sciences Politiques et Sociales de l'Université de Lausanne (Unil). Des versions préliminaires ont été présentées lors de deux colloques, qui se sont tenus en 2017 et 2018 54. Partant du constat que le renseignement constitue une entrée privilégiée pour étudier les relations dialectiques qu'entretiennent contestation et légitimation dans l'ordre politique, ce numéro double a pris le parti de croiser des recherches portant sur des époques et des pays différents. Peut-on en effet s'intéresser à l'institutionnalisation de la surveillance d'État sans prendre en compte son historicité ? Peut-on saisir les pratiques de ses protagonistes sans les insérer dans les échanges inter et intra-institutionnels au

50. Lascoumes P. et Le Bourhis J-P., « Des “passe-droits” aux passes du droit », art. cit., p. 56.

51. Bourdieu P., «Droit et passe- droit... », art. cit., p. 89.

52. Comme en témoigne largement la contribution récente dans cette revue de HennetteVauchez S., «La fabrique législative de l'état d'urgence : lorsque le pouvoir n'arrête pas le pouvoir ", Cultures E Conflits, n 113, 2019, pp. 17-41.

53. https://www.sciencespo.fr/ceri/fr/content/usages-des-technologies-liees-linterception-descommunications-utic. Consulté le 24 novembre 2019.

54. «Le renseignement politique intérieur dans les démocraties libérales : entre légitimation et contestation », Faculté de Sciences Politiques et Sociales de l'Université de Lausanne (Unil), 8 et 9 décembre 2017 et «SIGINT Intelligence Transnational Activities in France and Europe : Transnationalisation, Oversight and the Role of Courts », CERI-Sciences Po, Paris, 24 et 26 septembre 2018. 
sein, et même en dehors, de l'État ? Comment comprendre l'activité ordinaire des surveillants sans porter attention aux répercussions (ignorance, sentiment d'impuissance, résistance, (dé)mobilisation, (dé)radicalisation, etc.) sur les populations spécifiquement surveillées ? Aussi, pour clarifier ces questions et y apporter des éléments de réponse, mieux vaut-il inscrire sa perspective dans une optique relationnelle et processuelle : ce sont des relations, notamment des relations de domination, elles-mêmes liées à des processus sociohistoriques (la construction des États-nations, les guerres mondiales, la guerre froide, la décolonisation, etc.) qu'il faut expliquer. Les différentes contributions s'efforcent donc de situer et d'analyser ces relations changeantes entre différents types d'acteurs. À rebours d'approches réifiant " des contextes », qui font office de boîte noire, la prise en compte des réseaux d'interdépendances tissés par ces relations permet de penser en termes de configurations, comme y invitait Norbert Elias 55.

Dans ce numéro, il s’agit également de réfléchir ensemble aux façons de construire le renseignement en objet de recherche étayé par des matériaux empiriques. La vaste littérature académique sur le renseignement reste - à de notoires exceptions près 56 - dominée par les «témoignages » de professionnels du secteur et par des essais de journalistes, d'universitaires et autres commentateurs, consistant pour l'essentiel à dénoncer ou à célébrer l'activité des services spécialisés. La réduction quasi systématique des scandales qui rythment l'histoire du renseignement à l'occurrence de faits supposés scandaleux accentue cette emprise des positionnements normatifs. Dans ces conditions, où l'enjeu consiste surtout à justifier ou à condamner telle ou telle pratique, la mise à distance afférente à l'objectivation s'avère pour le moins compliquée. Loin du plaidoyer pro domo ou de la charge contre la surveillance, il s'agit d'abord et avant tout de normaliser et de désingulariser cet objet en refusant de le mythologiser et de le pathologiser, ce que la prégnance des fictions (littérature, cinéma, séries) et la virulence des polémiques rendent difficile. Face à la tentation de ne livrer que son opinion, la durée et la qualité de l'investigation peuvent faire figure de garde-fou. Aussi ont été réunis des points de vue pluriels quant à leur démarche et leurs méthodes, mais tous informés par une solide enquête empirique. Comme le montrent les contributions, à l'encontre d'un lieu commun savant, ce ne sont pas les sources qui manquent en la matière : archives (publiques et privées), entretiens (lorsque la proximité dans le temps le permet), observation directe (certes compliquée en ce domaine, mais cela n'a rien d'exceptionnel), publications officielles, correspondance,

55. Elias N., Qu'est-ce que la sociologie ?, Paris, Presses-Pocket, 1993, pp. 154-161.

56. En français, on mentionnera notamment Berlière J-M., Le monde des polices en France, Bruxelles, Complexe, 1996 ; Dewerpe A., Espion..., op. cit. ; Laurent S., Politiques de l'ombre. État, renseignement et surveillance en France, Paris, Fayard, 2009 et Rios-Bordes A., Les savoirs de l'ombre. La surveillance militaire des populations aux États-Unis (1900-1941), Paris, Éditions de l'EHESS, 2018. 
mémoires, presse et littérature secondaire offrent aux chercheurs en sciences sociales de nombreuses opportunités de questionner les ressorts de la légitimation et de la contestation du renseignement.

Première contribution à ce numéro, l'article de Jean-Marc Berlière entend soumettre à l'enquête historique l'énigme que pourrait constituer le maintien d'une police politique sous la III République, alors même que les républicains avaient envisagé sa suppression en 1870, l'estimant incompatible avec le respect des libertés publiques propre à un régime démocratique. C'est donc bien la question de la légitimation du renseignement politique intérieur (RPI) que l'historien, à partir d'une fréquentation au long cours des archives de police, entend prendre pour objet, en proposant toutefois un déplacement de la perspective d'analyse. Mettant à distance une représentation « complotiste » de la police politique (celle de dénonciateurs enclins à la parer d'un pouvoir exorbitant ou à lui imputer toutes sortes de pratiques occultes aussi bien qu'illégales et antidémocratiques), il propose d'étudier la façon dont les services de « haute police » se sont institutionnalisés, notamment par leur contribution à la neutralisation de la «menace anarchiste » ou de la poussée nationaliste, pendant et après la crise boulangiste. Partant de l'ordinaire des services et du travail au quotidien des agents (structures, moyens, organisation), il analyse leurs pratiques, routines et méthodes, une manière sans doute pertinente d'envisager les controverses suscitées par la police politique "républicaine », oxymore ou nécessité selon les acteurs, les cibles et les conjonctures historiques, mais aussi dimension centrale de l'exercice du pouvoir régalien.

Si la légitimation du renseignement réside pour beaucoup dans les soutiens dont il bénéficie au sein d'autres institutions dont la coopération fait l'État, elle repose aussi sur la manière dont les agents se sentent plus ou moins autorisés et motivés à prendre pour cible telle ou telle frange de la population. S'appuyant sur des archives de la préfecture de Police de Paris et du ministère de l'Intérieur, Magali Della Sudda analyse comment des femmes catholiques de la grande bourgeoisie conservatrice, gardiennes de l'ordre social, en sont venues à être considérées comme des ennemies de la III République. Liée à l'affirmation de la laïcité, à la séparation des Églises et de l'État, mais aussi à l'affaire Dreyfus, cette transformation des catégories de l'action étatique s'inscrit dans la difficile consolidation du régime républicain. Aux yeux des agents de renseignement, nombre d'éléments justifient la surveillance des dirigeantes de la Ligue des femmes françaises et de la Ligue patriotique des Françaises, qui, en 1914, revendiquent 100000 et 500000 adhérentes. Ces femmes semblent faire irruption dans le jeu politique pour soutenir les partis réactionnaires et antirépublicains. En outre, leur proximité avec le clergé en fait des alliées d'une puissance étrangère, le Vatican. Ces accointances sont donc étroitement observées, via des informateurs, en ce qu'elles passent pour déloyales, voire séditieuses. Des schèmes classiques d'analyse de la subversion sont ici 
redoublés par des stéréotypes de genre qui présentent tour-à-tour ces femmes comme manipulées (en raison d'un manque de libre-arbitre qui serait inhérent à la condition féminine) ou comme manipulatrices (par les pressions intimes qu'elles pourraient exercer sur les « hommes tièdes »).

En régime démocratique, l'institutionnalisation et l'extension du renseignement semblent contrevenir au pacte de confiance censé unir l'État à son peuple. C'est un problème politique qu'Alexandre Rios Bordes examine par une approche sociohistorique des services secrets de l'Armée et de la Marine aux États-Unis durant la première moitié du XXe siècle. Après avoir dépouillé des archives militaires comprenant des mémorandums, rapports, correspondances et missives, il montre comment dirigeants et agents de la Military Intelligence Division et de l'Office of Naval Intelligence n'auront eu de cesse de s'arranger avec ce qui pourrait être vécu comme une contradiction dérangeante entre la célébration officielle des libertés d'expression des citoyens et l'immixtion croissante des forces armées dans les affaires civiles. C'est surtout avec la Première Guerre mondiale et au nom de la « défense nationale » que le contrôle des "éléments hostiles ou potentiellement hostiles » devient un impératif au sein de ces deux services du renseignement militaire. Cette tentative de maîtrise du front intérieur, qui va se perpétuer et même s'intensifier après le conflit, fait de la population une problématique militaire. Inavouable publiquement, cette surveillance de plus en plus intrusive des individus et groupes jugés subversifs génère des tensions au sein même des services, mais c'est en liant menaces intérieures et menaces extérieures, une continuité forgée par le concept de « sécurité nationale », que cet accommodement va être justifié parmi les effectifs surveillants, forme de dissonance cognitive renforcée par la Seconde Guerre mondiale, la guerre froide puis la guerre contre le terrorisme.

L'existence d'une police politique ne va donc pas sans tensions en contexte démocratique. Dans le cas de la Suisse durant la guerre froide étudié par Fabien Thétaz, la police politique, sous l'appellation à la fois commune et officielle de «protection de l'État » (Staatsschutz), est hautement révélatrice des enjeux propres à la domination étatique. Son existence ne constitue pas un secret absolu, mais ses activités demeurent floutées par l'ensemble des autorités fidèles à la pratique du «chèque en gris ». À partir de documents officiels, d'articles de presse et d'entretiens, il retrace comment le renseignement intérieur s'est consolidé au sortir de la Seconde Guerre mondiale autour d'une figure de l'ennemi intérieur incluant progressivement l'ensemble des forces de gauche, des parlementaires aux sympathisants. Quand bien même le Parti socialiste participe au gouvernement entre 1943 et 1953, puis sans interruption depuis 1959, ce ciblage est largement soutenu par les policiers, acquis aux mots d'ordre des dirigeants politiques, militaires et économiques qui dominent l'État fédéral. La surveillance des éléments subversifs figure d'ailleurs en 
bonne place dans le Manuel de défense civile édité en 1969 par le ministère de la Justice et diffusé à l'ensemble de la population, invitée à concourir à la "protection de l'État ». Les polémiques sur la légitimité de ce livret ouvrent une mise en cause plus explicite de la police politique dans les années 1970 tant par des élus de gauche que par des militants des « nouveaux mouvements sociaux ». Rapidement désamorcées, ces dénonciations publiques récurrentes indiquent à la fois l'ampleur de la surveillance et celle des collusions dont elle bénéficie chez des élites d'État.

Ce rapport de forces où les activités de renseignement intérieur paraissent, aux yeux mêmes de leurs principales cibles, difficilement scandalisables va brusquement changer durant l'hiver 1989-1990, parallèlement à l'effondrement des États membres du Pacte de Varsovie, avec l'irruption du «scandale des fiches ». Se basant sur des sources de presse et des publications des autorités fédérales (directives gouvernementales, rapports et débats parlementaires, arrêts du Tribunal fédéral, etc.), Hervé Rayner et Bernard Voutat abordent cet événement, qui voit trois cent mille citoyens réclamer l'accès aux fichiers de la police politique, comme une succession de séquences scandée par de fortes variations de la jouabilité de la dénonciation. Soudain portée par de puissantes mobilisations, la stigmatisation de ce que les protestataires nomment « l'État fouineur » prend de court les professionnels du renseignement. Cette concaténation de révélations, d'accusations et de revendications déstabilise les représentants des autorités fédérales, poussés à mettre en place toute une série de ripostes institutionnelles (ouverture des fichiers, mise à pied de dirigeants policiers, Commission d'enquête parlementaire sur le ministère de la Défense, etc.) débouchant sur un réagencement des règles, frontières et ressources entre les institutions politiques. Autre effet émergent, ce scandale contribue à une juridicisation du renseignement à travers l'activation de catégories juridiques de perception et de normes légales censées définir tant ses pratiques que les mécanismes de son contrôle politique, parlementaire, judiciaire ou administratif.

La légitimation d'un nouveau service de renseignement ne va pas de soi. $\mathrm{Si}$ l'expansion et la spécialisation du secteur ainsi que la multiplication des traces numériques ouvrent des possibilités, le droit d'entrée reste coûteux, les impétrants devant faire leurs preuves auprès des autres services, soit autant de concurrents que d'alliés potentiels. À partir d'une enquête par observation directe et par entretiens, Anthony Amicelle retrace le parcours de Fintrac, l'agence canadienne de renseignement financier, depuis sa création en 2000, à un moment où les services de renseignement financier se multiplient de par le monde. Pour ses fondateurs, il s'agit certes de débusquer et de recenser des flux de capitaux illicites, mais l'élaboration d'une banque de données doit d'abord et avant tout faciliter l'accréditation comme partenaire légitime par les autres agences de renseignement et par les services de police. Usant de 
schèmes de perception empreints d'économicisme, les agents aiment se présenter comme des « courtiers en données ». Cependant, c'est tout un ensemble de ressources, de savoirs et de savoir-faire, et notamment de capitaux (capital social via un réseau d'informateurs et de partenaires, informationnel par une base de données, culturel par la capacité d'analyse), qui rend possible cette accumulation d'une réputation, d'un capital symbolique, accordant à Fintrac, dont la position demeure subalterne, la légitimité à produire du renseignement au nom de la sécurité nationale.

Même s'il n'y a rien d'automatique en la matière, l'évolution des pratiques et de l'emprise des services de renseignement doit donc être mise en lien avec leurs usages divers et variés des innovations technologiques (et pas seulement avec les potentialités de ces dernières, comme fait un peu rapidement par certains auteurs, même critiques). Didier Bigo et Laurent Bonelli soulignent que la révolution numérique s'accompagne bel et bien d'une extension du domaine de la surveillance. D’abord pensées comme des opportunités commerciales par les fournisseurs privés, les traces laissées par les activités on line des individus peuvent devenir des données utilisées à des fins de renseignement. À partir d'entretiens menés pendant une vingtaine d'années auprès d'une centaine de professionnels et d'une analyse des correspondances multiples (ACM) portant sur vingt-cinq services appartenant à neuf États, les Five Eyes Plus (USA, Grande-Bretagne, Canada, Australie, Nouvelle-Zélande, Allemagne, France, Espagne, Suède), ils construisent un espace du renseignement. Celui-ci se distribue autour de trois pôles, qui rassemblent les services au-delà des frontières nationales, en fonction de leurs usages différenciés de la technologie et des finalités qu'ils poursuivent (de l'antiterrorisme à l'espionnage diplomatique ou commercial). À rebours d'une vision statocentrée de la " sécurité nationale ", cette enquête dresse ainsi les contours des solidarités transnationales de métier qui se sont forgées ainsi que des logiques et des rationalités spécifiques qui en constituent les soubassements.

Ce dossier se clôt par un texte de Barthélémy Michalon et Benjamin Puybareau, qui présentent les entretiens menés auprès de deux journalistes spécialistes des services de renseignement, Jean Guisnel, qui vient d'écrire une Histoire secrète de la DGSE (La Découverte, 2019) et Jacques Follorou, auteur de L'État secret, la démocratie mise à mal (Fayard, 2018). Leurs propos, et notamment les précisions qu'ils comportent sur leur rapport ambivalent à leurs sources et, partant, au secret, éclairent la façon dont ils peuvent nouer, sur la durée, des liens de confiance, voire de confidence, avec des interlocuteurs eux-mêmes intéressés aux dynamiques de légitimation et de contestation du renseignement.

Enfin, ce numéro de Cultures E Conflits contient également un Forum intitulé «Arpenter les territoires du secret». Il rassemble des textes plus 
courts issus d'une journée d'étude («Les territoires du secret : les sciences sociales face aux pratiques de confidentialité dans les mondes de la sécurité ») organisée par Emmanuel-Pierre Guittet et Julien Pomarède le 24 mai 2018 à l'Université libre de Bruxelles (ULB). Gregory Daho revient de manière réflexive sur sa recherche au sein du milieu militaire en France et en particulier sur ses entretiens avec les enquêtés. Sur cette même relation d'enquête, Christian Lequesne rappelle combien les universitaires disposent de moyens pour intéresser les différents types d'acteurs de la sécurité. Elspeth Guild prend pour objet les ressorts du programme étatsunien de détentions et de transferts illégaux et les fuites, en provenance de la CIA, qui ont facilité sa mise en cause. Enfin, Anthony Amicelle et Jean Bérard étudient comment la mise en équivalence du principe du secret bancaire et de celui de la vie privée a pu servir d'argument opposable à ceux qui, au nom de la sécurité nationale, exigent une restriction de l'opacité des flux financiers.

Confronté aux difficultés pratiques d'enquêter sur le monde du renseignement, le politiste britannique Peter Gill expliquait dans un ouvrage désormais classique, qu'écrire sur le sujet pourrait être comparé « à essayer de réaliser un grand puzzle de scie sauteuse quand l'image sur la boîte est incomplète, que vous avez seulement une partie des morceaux dont vous avez besoin et que certains d'entre eux peuvent tout à fait être ceux d'un autre puzzle 57 ». Pour mener à bien cette tâche, il invitait à garder en tête que les services de renseignement font partie des administrations d'État et qu'à ce titre, ils tendent pour l'essentiel à fonctionner comme elles. Il proposait donc de transposer les connaissances des sciences sociales acquises sur ces terrains pour comprendre des fonctionnements et des activités couverts par le secret, y compris dans leurs spécificités. Les différentes contributions rassemblées ici suivent cette ligne de pente. Loin de plaider pour la constitution de savoirs spécialisés, elles replacent au contraire l'étude de ces services dans une sociologie plus générale de l'administration, de l'État et de l'international. Et en étudiant comment s'articulent, dans des configurations historiques et nationales différentes, des dynamiques de contestation et des processus de légitimation, elles ont l'ambition d'apporter quelques pièces supplémentaires à un puzzle plus vaste encore, celui du fonctionnement de l'ordre social et politique en démocratie.

57. Gill P., Policing Politics. Security Intelligence and the Liberal Democratic State, London, Frank Cass Editor, 1994, p. 35. 